\title{
Host Plants of the Carpenter Bee, Xylocopa brasilianorum $L$. (Hymenoptera: Apoidea) in Puerto Rico ${ }^{1}$
}

\author{
George C. Jackson and Roy O. Woodbury²
}

\begin{abstract}
The host plants of the carpenter bee, cigarrón, Xylocopa brasilianorum L., whose flowers supply adult foods, nectar, and pollen, and whose woods are used as nesting sites are reported. More than ten years of observations and accumulated notes indicate that more than 284 dicotyledonous and one monocotyledonous species (male bamboo, Lendrocalamus strictus (Roxb.) Nees) are used for food. Of these 285 plants, 277 are reported for the first time for Puerto Rico. Thirteen species are used as nesting sites, with eight being first reports also. It is suggested that the carpenter bee inadvertently, directly or indirectly may be responsible for the pollination of 25 fruit and seed crops that are used as food by man in Puerto Rico.
\end{abstract}

\section{INTRODUCTION}

The purpose of this paper is to present some observations made on the biology of the carpenter bee, cigarron, Xylocopa brasilianorum L. (Fig. 1) in Puerto Rico. It is hoped that this report on the host plants that serve as nesting sites and their flowers which provide adult food (nectar and pollen) will add to our knowledge of the habits of our single speries of this widespread genus. It is also hoped that these additional data will stimulate research on this insect in the Caribbean Basin.

Dr. Agustin Stahl (20), is credited with the first reporting of Xylocopidea in Puerto Rico. In his collection of insular fauna, there were two specimens of $X$. morio Fab., one male (Cat. No. 58-97) and one female (Cat. No. 82-99). Gundlach (4) observed that the carpenter bee is very common in Puerto Rico, as it is in Cuba. He also noted the color differentiation between males (yellow) and females (black), and mentioned their wood nesting habits. Wolcott (21) lists the Puerto Rico species as $X$. brasilianorum L., based on determinations by S. A. Rhower, indicating that $X$. morio Fab. and $X$. aenipennis $\mathrm{L}$. are synonyms to $X$. brasilianorum L. He further reports adults nesting in dead trunks of

${ }^{1}$ Manuscript submitted to the Editorial Board, October 2, 1975. Parts of this paper were presented at the Annual Meeting of the Sociedad Puertorriqueña de Ciencias Agrícolas, Mayagüez Institute of Tropical Agriculture, Mayagüez, P.R. October 17, 1975

${ }^{2}$ Associate Horticulturist and Plant Taxonomist, Agricultural Experiment Station, respectively, Mayaguiez Campus, University of Puerto Rico, Río Piedras, P.R. 


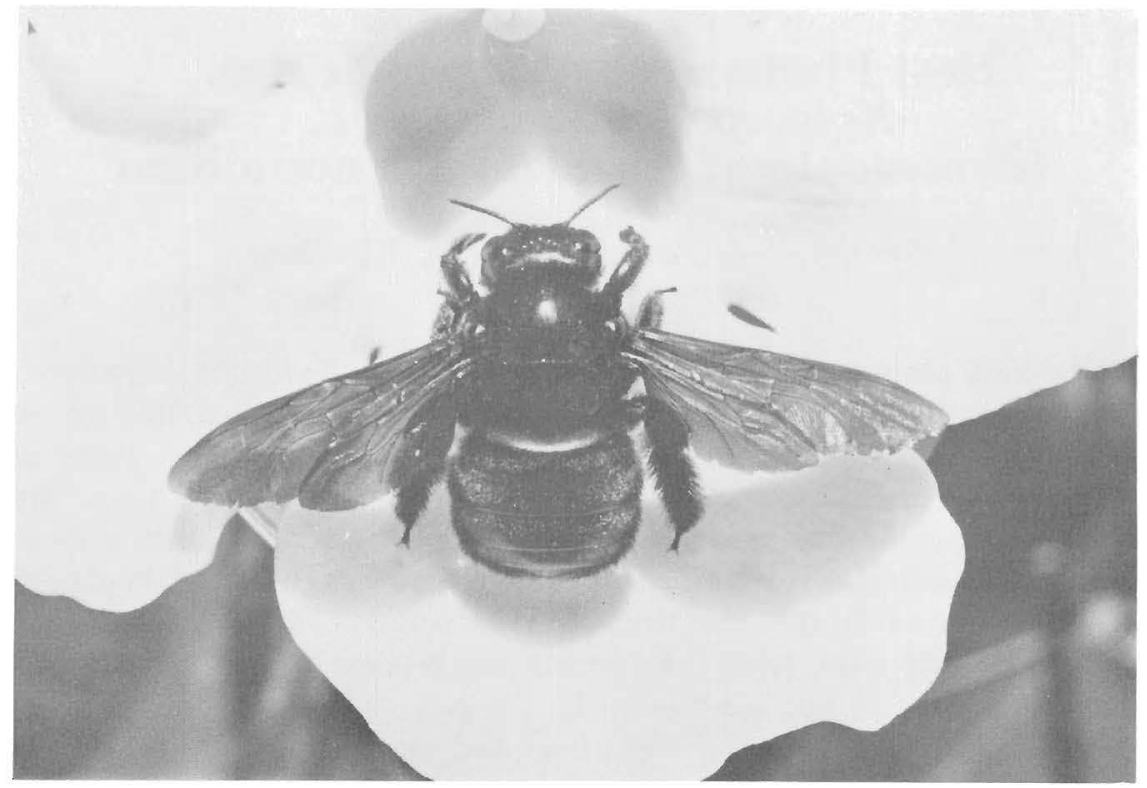

Fic. 1. - The Carpenter Bee Xylocopa brasilianorum L. on flower of the white clockvine, Thunbergia grandiflora Roxb. 'Alba'. Insect $1.6 \times$. (Photo G. C. Jackson.)

hibiscus, Hibiscus sp. at Río Piedras (24), and in fence posts at five different geographic locations (21, 22). Martorell (16) relates on those insects nesting in dead trunks and branches of the tall albizia, Albizia procera (Willd.) Benth., Ficus trigonata L. (F. stahlii Warb.) and West Indian mahogany, Swictenia mahagoni Jacq. in Puerto Rico. Wolcott $(23,24)$ and Ramos (19) report that this insect is abundant on Mona Island, and that they nast in Ficus trigonata L. (19) there. X. brasilianorum L. frequents many kinds of flowers $(16,24)$, and has been described specifically on Lantana sp.; sunflower, Helianthus annuus L. at Vega Alta and hibiscus, Hibiscus sp. at Río Piedras by Wolcott $(22,23,24)$.

The first report from Puerto Rico suggesting the pollinating activity of $X$. brasilianorum $\mathrm{L}$. was made by Martin et al. (15), working with Mexican species of Dioscorea. Jackson and Bauzá (5) demonstrated that the carpenter bee is a pollinator of the tropical nut, Lecythis elliptica Kunth. Barnés and Freyre (1) stated that this insect pollinates Tephrosia vogelii Hook. f.

\section{MA'TERIALS AND METHODS}

This study was started before January 1964, as an accumulative list. From the start, carpenter bees have been observed at all seasons of the year and in practically all of our geoclimatological regions. 
Flowering plants utilized by this insect as a food source have been noted as to genera, species, and location, only after absolute certainty of food collecting activity had been made. Common English $(2,7,11,12)$ and/or Spanish plant names $(2,11,12,18)$, when known, have been included. Location of the first observed feeding site is also listed. The taxonomic nomenclature of Britton and Wilson (2), as revised by Liogier $(9,10)$ is used.

Nests were not collected but only recorded as to genera and species of plant material, after their occupation by the carpenter bee was positive.

\section{RESULTS}

\section{GRAMINEA：BACIFFERAE}

Dendrocalamus strictus (Roxb.) Nees.

Myinwa flower, male bamboo, bambú, at Río Piedras.

$$
\text { PALMAÊ }
$$

Caryota mitis Lour.

Tufted fish-tail-palm, at Santurce.

Coccothrinax alta Beccari.

(C. argentea Lodd.) (Sargent), Puerto Rican silver-palm, palma plateada, at Florida.

Cocos nucifera L.

Coconut palm, palma de coco, at Santurce.

Chrysalidocarpus lutescens Wendl.

Yellow butterfly-palm, palma dorada, areca, at Santurce.

Gaussia attenuata (Cook) Beccari.

Puerto Rican Llume-palm, llume, at Vega Baja.

Hyophorbe verschaffeltii WendI.

Spindle-palm, at Río Piedras.

Livistonia chinensis $\mathrm{R}$. Br.

Chinese-fan-palm, palma de borbón, at Río Piedras.

Phoenix canariensis Chabaud.

Canary Island date palm, dátil, at Río Piedras.

P. dactylifera $\mathrm{L}$.

Date palm, dátil, at Ponce.

P. reclinata Jacq.

Cluster date palm, dátil, at Río Piedras.

P. roebelinii O'Brien.

Dwarf date palm, dátil, at Río Piedras.

Prestoea montana (R. Grah.) Nichols.

(Euterpe globosa Gaertn.), Mountain-palm, palma de sierra, at Toro Negro Mts. 
Pritchardia pacifica Seem \& Wendl.

Fiji Island fan-palm, palma de jardín, at Santurce.

P. thurstonii.

Thurstons pritchard-palm, at Santurce.

Roystonea borinquena O. F. Cook.

Royal-palm, palma real, at Fortuna.

R. regia (Kunth) O. F. Cook.

Cuban royal-palm, palma real cubana, at Río Piedras.

Sabal causiarum (Cook) Beccari.

Puerto Rican hat-palm, palma de sombrero, at Isabela.

Thrinax microcarpa Sarg.

Broom-palm, palma de escoba, at Punce.

Washingtonia robusta Wendl.

Washington-palm, palma del desierto, at Río Piedras.

AMARYLLIDACEAF.

Agave missionum Trel.

Century-plant, corita, at Juana Díaz.

A. sisalana Perrine.

Sisal agave, maguey, at Guánica.

Aloe vulgaris Lam.

Mediterranean aloe, sábila, at La Paraguera.

Furcraea tuberosa Ait. f.

Maguey criollo, female karata, at Salinas.

Hymenocallis americana Roem.

Lirio blanco, American hymenocallis, at Dorado.

Sansevieria laurenti

Congo snake, sansevieria, at Río Piedras.

Smilax coriacea Spreng.

Green-brier, bejuco de membrillo, at Tortugero.

DIOSCOREACEAE

Dioscorea composita Hemsl. (15)

Dioscorea, at Mayagüez.

D. floribunda Mart. \& Gal. (15)

Dioscorea, at Mayagüez.

D. spiculiflora Hemsl. (15)

Dioscorea, at Mayaguiez.

HAEMODORACEAE

Xiphidium coeruleum Aubl.

Mano de mono, at Río Piedras. 
ZINGIBERACEAE

Alpinia speciosa (Wendl.) K. Schum.

(Languas speciosa Small), Shell-flower, boca de dragón, at Corozal.

Costus macrostrobilus K. Schum.

Spiral-flag costus, caña india, at Luquillo mountains.

Hedychium coronarium Koenig.

Common ginger-lily, nardo, at Luquillo mountains.

Renealmia aromatica (Aubl.) Griseb.

(Alpinia aromatica Aubl.), Aromatic galingal, bihao, at El Verde.

R. exaltata L. f.

(Alpinia exaltata R. \& S.), Jengibre de jardín, at Bayamón.

Thalia geniculata L.

Thalia, pámpano, at Arecibo.

ORCHIDACEAE

Epidendrum ciliare L.

(Auliza ciliaris Salisb.), Birds-in-flight, dama de noche, at Guaynabo.

Vanda ("Miss Agnes Joaquim" Hookeriana Reichb.f. x V. teres Lindl. Vanda, orquídea vanda, at Guaynabo.

POLYGONACEAE

Antigonon leptopus Hook. \& Arn.

Mountainrose coral-vine, coralina, at Isabela.

A. guatemalense Meisn.

(A. macrocarpon Britt. \& Wil.), white coral-vine, coralina blanca, at Río Piedras.

Coccoloba costata Wr. $\times$ Sauv.

(Coccolobis rupicola Urban), at Vega Baja.

C. diversifolia Jacq.

(Coccolobis laurifolia Jacq.), Mixleaf seagrape, uvilla, at Bayamón.

C. Krugii Lindau.

Krug's seagrape, at Guánica.

C. microstachya Willd.

(Coccolobis obtusifolia Jacq.), Puckhout, uvillo, at Guánica.

C. pirifolia Desf.

At Florida.

C. pubescens L.

(Coccolobis grandifolia Jacq.), Grandleaf seagrape, moralón, at Florida. 
C. rugosa Desf.

Ortegón, at Guayanés.

C. Sintenisii Urban.

Uvero de monte, at Susúa forest.

C. swartzi Meisn.f. urbaniana (Lindl.) Howard.

(Coccolobis borinquensis Britt.), Ortegón, at Carite.

C. uvifera (L.) Jacq.

Common seagrape, uva de mar, at Santurce.

C. venosa $\mathrm{L}$.

Chiggery-grape, calambreña, at Fajardo.

Triplaris caracasana Cham.

Ant-tree, triplaris, at Río Piedras.

NYCTAGINACEAE

Boerhaavia scandens L.

Pega-pollo, at Guánica.

Bougainvillea spectabilis Willd.

Brazil bougainvilla, trinitaria, at Ponce.

Guapira discolor (Spreng.) Little.

Barrehorno, at Desecheo.

G. fragrans (Dum.-Cours.)

Black mampoo, corcho, at Guayama.

Mirabilas jalapa L.

Common four o'clock, siciliana, at Mayagüez.

Pisonia aculeata L.

Devil's-claw pisonia, escambrón, at Bayamón.

P. albida (Heimerl.) Britton.

Loblolly, palo bobo, at Guánica.

P. helleri Standley.

Hold-me-back, escambrón, at Sabana Hoyos.

P. subcordata Sw.

Loblolly, palo bobo, at Ramsey.

BASELLACEAE

Anredera leptostachys (Moq.) Steen.

(Boussingaultia leptostachys Moq.), Madeira-vine, suelda-consuelda, at Guayama.

MENISPERMACEAE

Cissampelos pareira L.

Velvet-leaf, bejuco de mono, at Ft. Buchanan. 
Magnolia grandiflora L.

Southern magnolia, magnolia, at Adjuntas.

M. portoricensis Bello.

Puerto Rican magnolia, jagüilla, at Mt. Guilarte.

M. splendens Urban.

Magnolia, laurel sabino, at Luquillo Mts.

LAURACEAE

Cinnamomum elongatum (Vahl.) Kosterm.

(Phoebe elongata (Vahl.) Nees), Avispillo, at Río Piedras.

Licaria brittoniana Allen \& Gregory.

Canela, at Maricao.

Ocotea floribunda (Sw.) Mez.

Ocotea, laurel, at Dorado.

Nectandra antillana Meisson.

Bedstead-wood, aguacatillo, at Dorado.

N. coriaceae (Sw.) Griseb.

Lance-wood, cigua, at Quebradillas.

N. Krugii Mez.

Laurel canelón, at Guavate forest.

N. patens (Sw.) Griseb.

Laurel roseta, at Florida.

Persea americana Mill.

(P. persea Cockrell), Avocado, aguacate, at Juana Díaz.

BRASSICACEAE

Brassica integrifolia (West) O. E. Schultz.

Wild mustard, mostaza, at Cayey.

CAPPARIDACEAE

Capparis cynophallophora L.

Jamaica caper, bejuco inglés, at Coamo.

C. flexuosa L.

Dog caper-tree, palo de burro, at Guánica.

C. indica (L.) Fawc. \& Rendle.

Liguam, at Fajardo.

Cleome spinosa Jacq.

Spiny spider-flower, flor de perro, at Salinas.

MORINGACEAE

Moringa oleifera Lam.

(M. moringa Millsp.), Horseradish-tree, ángela, at Salinas. 
Bryophyllum pinnatum (Lam.) Kurz.

Air-plant, bruja, at Tortuguero.

AMYGDALACEAE

Chrysobalanus Icaco L.

Coco-plum, hicaco, icaco, at Tortuguero.

LEGUMINOSAE: MIMOSOIDEAE

Acacia farnesiana (L.) Willd.

(Vachellia farnesiana Wight \& Arn.), Sweet-acacia, aroma, at Fajardo.

A. tortuosa (L.) Willd.

Twisted-acacia, aroma, at Cayo Muertos.

Albiza lebbeck (L.) Benth.

(Albizzia lebbeck (L.) Benth.), Woman's tongue, acacia amarilla, at Quebradillas.

Callinandra caracasana (Jacq.) Benth.

(Annesila portoricensis Britt.), white anneslia, cojobillo, at Hato Rey.

Inga fagifolia (L.) Willd.

(I. laurina (Willd.), pomshock, guamá, at Cayey.

I. vera Willd.

(I. inga Britt.), guaba-inga, guaba, at Cayey.

Pithecellobium dulce (Roxb.) Benth.

P. Saman (Willd.) Benth.

Guamachil ape's-earring, guamá Americano, at Río Piedras.

Raintree-saman, samán, at Río Piedras.

P. unguis-cati (L.) Benth.

Black-bead, uña de gato, at Guánica.

Prosopis juliflora (Sw.) D.C.

Mesquite, bayahonda, at La Parguera.

LEGUMINOSAE: CAESALPINOIDEA

Caesalpinia crista L.

(Guilandia crista (L.) Small), Gray nickers, mata de playa, at San Juan.

C. divergens Urban.

(Guilandia divergens (Urb.) Britt.), Small yellow nickers, mata de playa, at Luquillo Beach.

C. pulcherrima (L.) Sw.

(Poinciana pulcherrima L.), Barbados pride, clavellina, at Santurce. 
Cassia aeschynomene DC.

(Chamaecrista aeschynomene Greene), Partridge-pea, at Río Piedras.

C. antilliana (Britt. \& Rose) Alain.

(Chamaecrista antilliana Britt. \& Rose), Hediondilla, at Cayey.

C, bicapsularis L.

(Adipera bicapsularis (Britt. \& Rose), Christmas-bush, hoja de sen, at Farjado.

C. fistula L.

Golden shower senna, caña fístula, at Aguirre.

C. javanica L.

(C. nodosa Hamilt.), Appleblossom senna, cassia rosada, at Río Piedras.

C. polyphylla Jacq.

(Peiranisia polyphylla (Jacq.) Britt. \& Rose), Retama prieta, at Salinas.

C. stahlii Urban.

(Adiptera sthalii Britt. \& Rose), Stahl's cassia, vela muerto, at Cayey.

Delonix regia (Bojer) Raf.

Royal poinciana, flamboyán, at Cayey.

Haematoxylon campechianum L.

Logwood, campeche, at Río Piedras.

Hymenaea courbaril L.

West Indian locust, algarrobo, at Río Piedras.

Peltophorum inerme (Roxb.) Naves.

(P. ferrugineum (Dene.) Benth.), Yellow flamboyant, flamboyán amarillo, at Luquillo forest.

Poinciana gillesii Hook.

Paradise poinciana, at Santurce.

Tamarindus indica L.

Tamarind, tamarindo, at Jauca.

\section{LEGUMINOSAE: FABOIDEA.}

Cajanus cajan (L.) Millsp. (3).

(Cajan cajan Millsp.), Pigeon-pea, gandur, at Mayagüez.

Calopogonium coeruleum Benth.

Jicama, at Río Piedras.

Canavalia ensiformis (Jacq.) DC.

Sword-bean, haba de burro, at Río Piedras.

C. gladiata DC.

Horse-bean, at Río Piedras. 
C. maritima (Aubl.) DC.

Bay-bean, haba de playa, at Rincón.

Centrosema plumieri (Turp.) Benth.

(Bradburya plumieri Kuntze), Butterfly-pea, conchita, at Rio Piedras.

C. pubescens Benth.

(Bradburya pubescens Kuntze), Butterfly-pea, conchita, at Río Piedras.

C. virginianum Benth.

(Bradburya virginianum Kuntze), Butterfly-pea, conchita de Virginia, at Guaynabo.

Clitoria laurifolia Poir.

(Martiusia laurifolia Britt.), Pigeon-wings, conchita, at Tortuguero.

C. rubiginosa Juss.

(Martiusia rubiginosa Britt.), Pigeon-wings, conchita, at Dorado.

Crotalaria latifolia L.

Rattle-box, matraca, at Coamo.

C. retusa L.

Yellow lupin, matraca, at Aguirre.

C. saltiana Andr.

(C. striata DC.), Rattle-box, matraca, at Dorado.

C. verrucosa $\mathrm{L}$.

Blue rattle-box, matraca, at Vacía Talega.

Dalbergia ecastaphyllum (L.) Taub.

(Ecastophyllum ecastophyllum Britt.), Coin-vine, palo de pollo, at Dorado.

D. monetaria 1. f.

(Securidaca volubilis L.), Money-bush, membrillo, at Sabana Hoyos.

Erythrina eggersii Krug, \& Mold.

(E. horrida Eggers), Cock's-spur-coral-bean, espuela de gallo, at Río Piedras.

E. variegata L. 'occidentalis' (L.) Merrill.

Variegated coral-bean, bucayo haitiano, at Santurce.

Gliricidia sepium (Jacq.) Kunth ex Walp.

Madre de cacao, at Río Piedras.

Goeffrea inermis W. Wright.

(Andria inermis H.B.K.), Bastard-mahogany, moca, at Cayey.

Lablab niger Medic.

(Dolichos lablab L.), Lablab-bean, chícharos, at Tortuguero.

Machaerium lunatun (L.F.) Ducke

(Drepanocarpus lunatus (L.F.) G.F.W. Meyer), Escambrón, at Humacao beach. 
Macroptilium lathyroides (L.) Urban.

(Phaseolus lathyroides L.), Wild bush-bean, habichuela parada, at Luquillo beach.

Mucuna solanei Fawc. \& Rendle.

Sea-bean, ojo de buey, at Dorado.

Phaseolus adenanthus G.F.W. Meyer.

Wild-bean, habichuela cimarrona, at Guaynabo.

P. vulgaris L. 'Contender'.

Snap-bean, habichuela tierna, at Isabela.

Pitetia aculeata (Vahl.) Urban.

Fustic, tachuelo, at Coamo.

Pterocarpus indicus Willd.

India padunk, oreja de mono, lluvia de oro, at Río Piedras.

P. officinalis Jacq.

Swamp-bloodwood, palo de pollo, at Humacao.

Sabinea florida (Vahl.) DC.

Wattapama, retama, at Río Abajo forest.

Sesbania grandiflora (L.) Pers.

(Agati grandiflora Desv.), Agati sesbania, gallito, at Guánica.

Tephrosia vogelii Hook. F. (1).

Vogel tephrosia, tefrosia, at Mayagüez.

Vigna luteola (Jacq.) Benth.

(V.repens (L.) Kuntze), Cow-pea, at Guaynabo.

V. vexillata (L.) A. Rich.

Cow-pea, frijol cimarrón, at Guaynabo.

ZYGOPHYLLACEAE

Guaiacum officinale L.

Common lignum-vitae, guayacán, at San Juan.

G. sanctum L.

Holywood lignum-vitae, guayacán blanco, at Guánica.

\section{MALPIGHIACEAE}

Brachypteris ovata (Cav.) Small.

At Humacao.

Bunchosia glandulosa (Cav.) DC.

Café forastero, at Florida.

Byrsonima coriacea Millsp.

Maricao, at Tortuguero.

B. lucida (Sw.) DC.

(B. cuneata P. Wil.), Locust-berry, palo de doncella, at Sabana Grande. 
Malpighia glabra L.

Barbados-cherry, cereza, at Aguadilla.

M. punicifolia L.

West Indian-cherry, acerola, at Juana Díaz.

M. shaferi Britt. \& Wilson.

Olaga, at Maunabo.

Stigmaphyllon periplocifolium (Desf.) Juss.

(S. lingulatum Small), Amazon-vine, bejuco de San Pedro, at Santa Isabel.

S. tomentosum (Desf.) Ndz.

Amazon-vine, bejuco de toro, at Coamo.

RUTACEAE

Citrus medica L.

Citron, cidra, at Adjuntas.

Murrya exotica L.

(Chalcas exotica (L.) Millsp.), Orange jesamine, café de la India, at Fortuna.

BURSERACEAE

Bursera simaruba (L.) Sarg.

(Elaphrium simaruba (L.) Rose), West Indian-birch, gumbolimbo, at Coamo.

\section{MELIACEAE}

Melia azedarach L.

China-berry, lilaila, at Río Piedras.

POLYGALACEAE

Polygala cowellii (Britt.) Blake.

(Phlebotaenia cowellii Britt.), Violet-tree, violeta, at Coamo.

EUPHORBIACEAE

Aleurites moluccana (L.) Willd.

Candle-nut, nuez de la India, at Florida.

Croton discolor, Willd.

Lechecillo, at Guánica.

C. lucidus L.

Fire-bush, at Guánica.

C. rigidus (Muell. Arg.) Britton.

Yellow balsam, adormida, at Quebradillas.

Euphorbia pulcherrima Willd.

Poinsettia, flor de pascua, at Aibonito. 
Hura crepitans L.

Sand-box, javilla, at Río Grande.

Jatropha gossypifolia 1.

(Adenoropium gossypifolium Pohl.), Tuatúa, at Vacía Talega.

Jatropha integerrima Jacq.

(Adenoropium hastatum (Jacq.) Britt. \& Wilson), Peregrina nettlespurge, peregrina, at Santurce.

Pedilanthus tithymaloides (L.) Poit.

Slipper-plant, ipecacuana, at Santurce.

Ricinus communis L.

Castor-oil plant, higuereta, at Vacía Talega.

ANACARDIACEAE

Anacardium occidentale L.

Cashew-nut, cajuil, pajuil, at Tortuguero.

Schinus terebinthifolius Raddi.

Christmas-berry-tree, pimienta del Brasil, at Florida.

CELASTRACEAE

Cassine xylocarpa Vent.

(Elaeodendron xylocarpum DC.), Marble-tree, coscorrón, at Vacía Talega.

\section{HIPPOCRATEACEAE}

Hippocratea volubilis $\mathrm{L}$.

Bejuco prieto, at Sabana Hoyos.

Pristimera caribaea (Urb.) A.C.Sm.

(Hippocratea caribaea Urb.), at Sabana Hoyos.

DODONAEACEAE

Dodonaea viscosa Jacq.

(D. ehrenbergii Schl.), Varnish-leaf, chamiso, at Vacía Talega.

SAPINDACEAE

Serjania polyphylla (L.) Radlk.

Basket-wood, bejuco de costilla, at Vacía Talega.

RHAMNACEAE

Colubrina arborescens (Mill.) Sarg.

(C. colubrina Millsp.), Soap-tree, abejuelo, at Vacía Talega.

Gouania lupuloides (L.) Urban.

Chew-stick, bejuco de sopla, at Vacía Talega.

Ziziphus jujuba)L.) Lam.

Jujube, aprín, at Fajardo. 
Z. reticulatus (Vahl.) DC.

(Sarcomphalus reticulatus (Vahl.) Urb.), Espejuelo, at Guánica.

MALVACEAE

Abelmoschus esculentus (L.) Moench.

Okra, quimbombó, at Santa Isabel.

Hibiscus (L.) spp. (15).

Hibiscus, pavona, at Rio Piedras.

H. bifurcatus Cav.

Fork-leaf hibiscus, buenas tardes, at Tortuguero.

H. forcellatus Lam.

At tortuguero.

H. rosa-sinensis L.

Chinese hibiscus, amapola, at Fortuna.

H. trilobus Aubl. Pl.

At Tortuguero.

H. sabdariffa L.

Jamaica-sorrel, viña, at Santurce.

H. schizopetalus (Mart.) Hook.

Fringed-hibiscus, lira, at Santurce.

Malache spinifex (L.) Kuntze.

Barb-fruit pavonia, cadillo espinoso, at Quebradillas.

Malachra alceifolia Jacq.

Bastard-okra, malva de caballo, at Santurce.

STERCULIACEAE

Helicteres jamaicensis Jacq.

Cow-bush, huevo de gato, at Tortuguero.

Melochia tomentosa L.

(Moluchia tomentosa (L.) Britt.), Broom-wood, bretónica afelpada, at Santa Isabel.

Melochia villosa (Mill.) Fawc. \& Rendle.

(Riedlea hirsuta DC.), Bretónica peluda, at Tortuguero.

OCHNACEAE

Ochna multiflora DC.

Ochna, at Guaynabo.

Oratea littoralis Urban.

Abeyuelo, at Tortuguero.

GUTTIFERAE

Garcinia dulcis (Roxb.) Kurz.

Mangosteen, mangostán, at Río Piedras. 
G. tinctoria (DC.) Dunn.

Garcinia, mangostín, at Río Piedras.

Mammea americana L.

Mammy-apple, mamey, at Maunabo.

FLACOURTIACEAE

Casearia decandra Jacq.

Wild-honey-tree, gía verde, at Río Piedras.

Homalium racemosum Jacq.

Caracolillo, at Monte del Estado.

PASSIFLORACEAE

\section{Passiflora foetida L.}

Love-in-the-mist, tagua-tagua, at Santa Isabel.

P. laurifolia L.

Yellow-granadilla, parcha, at Guaynabo.

\section{CACTACEAE}

Consolea rubescens (Salm-Dyck. ex DC.) Lam.

(Opuntia rubescens (Salm-Dyck), Prickly-pear, tuna de yagua, at Guánica.

Hylocereus trigonus (Haw). Stafford.

Night-blooming cereus, pitajaya, at Dorado.

Lamaireocereus hystrix (Haw.) Britt. \& Rose.

Spanish-dildo, dildo español, at Guánica.

Opuntia dillenii (Ker-Gawl) Haw.

Dillen prickly-pear, tuna brava, at Guánica.

Pilosocereus royenii (L.) Byles \& Rowley.

(Cephalocereus royenii (L.) Britt. \& Rose), Royen pilocereus, sebucán, at Guánica.

\section{LYTHRACEAE}

Cuphea platycentra Lem.

(Parsonsia platycentra (Lem.) Britt.), Cigar-flower, chiagari, at Guaynabo.

Lawsonia inermis L.

Henna-plant, resedá, at Dorado.

PUNICACEAE

Punica granatum L.

Pomegranate, granada, at Salinas. 
Couroupita guianesis Aubl.

Cannon-ball tree, bala de cañón, at Río Piedras.

Lecythis elliptica Kunth (5).

Monkey-pot, nuez de paraíso, at Juana Díaz.

COMBRETACEAE

Buchenavia capitata (Vahl.) Eichl.

Granadillo, at Tortuguero.

Terminalia catappa L.

Indian-almond, almendrán, at Isla Verde.

Laguncularia racemosa (L.) Gaertn.

White-mangrove, mangle blanco, at Aguirre.

MYRTACEAE

Eugenia jambos (L.) Millsp.

Rose-apple, pomarrosa, at Tortuguero.

E. malaccensis L.

Malay-apple, manzana malaya, at Río Piedras.

Psidium guajava L.

Guava, guayaba, at Juana Díaz.

EBENACEAE

Diospyros philippensis (Desr.) Guercke.

(D. discolor Willd.), Velvet-peach, mabolo, at Río Piedras.

SYMPLOCACACEAE

Symplocos martinicensis Jacq.

Martinique sweet-leaf, aceituna blanca, at Tortuguero.

OLEACEAE

Syringa vulgaris L.

Common lilac, at Guayama.

APOCYNACEAE

Nerium oleander L.

Oleander, adelfa, at Guaynabo.

Vinca rosea $\mathrm{L}$.

Madagascar periwinkle, playera, at Aguirre.

CONVOLVULACEAE

Ipomoea batatas (L.) L. (3).

Sweet-potato, batata, at Mayagüez. 
I. carnea Jacq.

Morning-glory, batatilla carnosa, at Salinas.

I. cathartica Poir.

Morning-glory, bejuco de gloria, at Tortuguero.

I. crassicaulis (Benth.) Robinson.

Aguinaldo morado, at Pastillo.

I. pes-caprae (L.) Roth.

Soil-bind morning-glory, bejuco de playa, at Vacía Talega.

I. quamoclit L.

(Quamoclit quamoclit Britt.), Cypress-vine, cambustera, at Isabela.

I. rubra (Vahl.) Millsp.

Morning-glory, bejuco de puerco, at Tortuguero.

I. stolonifera (Cyrill) Poir.

Beach morning-glory, bejuco de costa, at Salinas.

I. tiliacea (Willd.) Choisy.

Morning-glory, bejuco de puerco, at Tortuguero.

Merremia dissecta (Jacq.) Hall f.

(Ipomoea dissecta Pursh), Noyau-vine, noyo, at Tortuguero.

Porana paniculata Roxb.

Christmas-vine, coralita blanca, velo de novia, at Río Piedras.

Stictocardia campanulata (L.) Millsp.

Morning-glory, at Manatí.

Turbina corymbosa (L.) Raf.

Christmas-vine, aguinaldo, at Santurce.

VERBENACEAE

Citharexylum fruticosum L.

Fiddle-wood, péndula, at Tortuguero.

Clerodendrum aculeatum (L.) Schlect.

(Volkameria aculeata L.), Prickly-myrtle, escambrón blanco, at

Vacía Talega.

Duranta repens L.

Golden-dew drop, lila, at Río Piedras.

Latana spp. (22).

Lantana odorata L.

Button-sage, Santa María, at Santurce.

Tectona grandis L. F.

Teak, teca, at Río Piedras.

Vitex angus-castus L.

Chaste-tree, suazgatillo, at Río Piedras.

LABIATAE

Coleus Blumei Benth.

Common coleus, vergüenza, at Carolina. 
Hyptis atrorubens Poit.

Bush-mint, marrubio, at Dorado.

H. capitata Jacq.

Wild-hops, marrubio obscuro, at Dorado.

H. lantanifolia Poit.

Bush-mint, marrubia blanco, at Dorado.

Leonotis nepetaefolia (L.) R.Br.

Lion's-ear, molinillo, at Rio Piedras.

Leonurus sibiricus L.

Lion's-tail, agripalma, at Río Piedras.

Ocimum basilicum L.

Sweet-basil, albahaca, at Santa Isabel.

O. sanctum L.

Mosquito-plant, albahaca, at Manatí.

SOLANACEAE

Brunsfelsia americana L.

Franciscan rain-tree, aguacero, at Guayama.

B. undulata Sw.

Lady-of-the-night, alelí, at Santurce.

Dunalia arborescens (L.) Sleumer.

Galán arbóreo, at Cayey.

Lycopersicon esculentum Millsp. 'Manapal'.

Tomato, tomate, at Río Grande.

Nicotiana tabacum L.

Tobacco, tabaco, at Guayama.

Solandra grandiflora Sw.

(Swartzia grandiflora Gmel.), Swartz-pea, bejuco de pedro, at Santurce.

Solanum melongena $\mathrm{L}$.

Eggplant, berejena, at Santa Isabel.

S. seaforthianum Andr.

False-belladonna, jazmin de Italia, at Guaynabo.

S. wendlandii Hook. F.

Costa Rican nightshade, campana de pascua, at Guayama.

SCROPHULARIACEAE

Agalinis fasciculata (Ell.) Raf.

Gerardia, yerba verónica, at Tortuguero.

BIGNONIACEAE

Cydista aequinoctialis (L.) Miers.

Guard-withe, bejuco blanco, at Dorado. 
Distictis lactiflora (Vahl.) DC.

Vanilla-distictis, liana fragante, at Salinas.

Doxantha unguis-cati (L.) Rehder.

(Batocydia ungis (L.) Mart.), Cat-claw, pega palo, at Salinas.

Enallagma latifolia (Mill.) Small

Black-calabash, higüerillo, at Vega Baja.

Jacaranda acutifolia H. \& B.

Sharp-leaf jacaranda, flamboyán azul, at Guaynabo.

Macrocatalpa longissima (Jacq.) Britt.

Haiti-catalpa, at Mayagüez.

Podranea ricasoliana Sprague.

Ricasoliana, millonaria, at Guayama.

Spathodea campanulata Beauv.

African tulip-tree, tulipán africano, at Dorado.

Tabebuia argentea (Bur. \& Schum.) Britt.

Silver trumpet-tree, roble amarillo, at Río Piedras.

T. glomerata Urban.

Yellow poui, roble amarillo, at Bayamón.

T. haemantha (Bert). DC.

Roble colorado, at Susúa forest.

T. heterophylla (DC.) Britt.

Pink trumpet-tree, roble blanco, at Santurce.

Tecoma stans (L.) H.B.K.

Ginger-thomas, roble amarillo, at Ponce.

ACANTHACEAE

Asytasia gangetica T. Anders.

Creeping thunbergia, at Aguirre.

Thunbergia erecta T. Anders.

Bush clock-vine, angelina, at Río Piedras.

T. grandiflora Roxb.

Bengal clock-vine, pompeya, at Guaynabo.

T. grandiflora Roxb. 'Alba'.

White clock-vine, at Guaynabo.

RUBIACEAE

Ixora coccinea L.

Burning-love, cruz de Malta, at Río Piedras.

CUCURBITACEAE

Cucumis sativus L. 'Ashley'.

Cucumber, pepinillo, at Río Grande. 
Cucurbita moschata (Dutch.) D. \& P.

(Pepo moschata Britt.), Pumpkin, calabaza, at Guaynabo.

Luffa cylindrica (L.) Roemer

Vegetable sponge, esponja. At Arús.

COMPOSITAE

Helianthus annuus L. "Mammoth Grey Stripe."

Sunflower, girasol, at Vega Alta (23).

Senecio confusus Britt.

Mexican daisy, margarita, at Mayagüez.

Tithonia diversifolia (Hemsl.) Gray

Mexican sun-flower, girasol mexicano, at Aguirre.

\section{NESTING SITES}

Plants or plant material observed as nesting sites are as follows: Dead trunks of treefern, Alsophila or Cyathea spp., at Guaynabo; redwood fencing, Sequoia semperiverens Endl., at Aguirre; deadwood of poinsettia, Euphorbia pulcherrima Willd., at Ponce; dead trunks of hogplum, Spondias mombin L., at Santurce; dead branches of royal poinciana, Delonix regia Raf., at Hato Rey; stumps of logged teak, Tectona grandis L.f., at Río Abajo; also in fence posts of bucare, Erythrina spp., and West Indian Birch, Bucera simaruba (L.) Sarg., at Caonillas and Loíza, respectively.

\section{DISCUSSION}

The 285 listed food plant species belonging to 189 genera and 57 families represent a rather wide spectrum of our insular flora. By no means is it considered complete. All of the listed food plant species are dicotyledonous, with exception of one, the male bamboo, Dendrocalamus strictus (Roxb.) Nees (14), ${ }^{3}$ Of these 285 plants, 277 are first reports for Puerto Rico. The fruit or seeds of 25 or more of these reported plants, such as avocado, Barbados cherry, cashew, citron, coconut, cucumber, eggplant, guava, icaco-plum, malay-apple, mamme-apple, mangosteen, okra, passion-fruit, pigeon-pea, pomegranate, pumpkin, rose-apple, sea-grape, snap-bean, sorrel, sunflower, tamarind, tomato, velvet-peach, and West Indian cherry are food sources of man. The possibility should not be overlooked that the female carpenter bee, while collecting nectar and pollen, may inadvertently be directly or indirectly responsible for their pollination.

\footnotetext{
${ }^{3}$ Other Hymenoptera observed feeding on D. strictus (Roxb.) Nees were: the honey bee, Apis mellifera L., paper-nest wasps, Polistes americanus Fab. and P. major Palisot de Beauvois.
} 
More than 50 fruit and seed crops of the world depend upon insect pollination for satisfactory yields (8). The ability of the carpenter bee as a pollinator of Dioscorea (15), Lecythis (5), and Tephrosia (1) has been mentioned. Xylocopidea other than $X$. brasilianorum L. are recognized as pollinators in other world areas. Lindsey (8) cites records of Xylocopa spp. pollinating jute, Corchorus capsularis L. and sunn hemp Crotalaria juncea L., in India. In Hawaii (17), a single species is one of the most important pollinators of passion-fruit, Passiflora edulis L. and $P$. flavacarpa Degener. Two species in India (6) are pollinators of Luffa spp.

$X$. brasilianorum L. appears chiefly to utilize a variety of dead, and systematically unrelated plants or plant derivatives for nest construction, with her preferences being for soft to moderately hard woods. Of the eight plants listed as nesting sites, six are trees. The woods of four locally grown species are rated from very soft for ceiba to moderately hard for teak, and with specific gravity ranging from 0.23 for ceiba, to 0.8 for royal poinciana (13).

In general, the carpenter bee's continued nesting in dead branches and stumps of trees is of little or no economic importance. Her value as a pollinator of native and introduced food plants probably offsets the incidental damage caused by her nesting within structural timbers.

\section{RESUMEN}

Notas acumuladas durante más de diez años de observaciones indican que el cigarrón utiliza unas 285 especies (una de ellas bambú) de plantas como fuente de alimento. De éstas, 277 se informan por primera vez en Puerto Rico. El cigarrón anida en trece especies, nueve de las cuales se mencionan como tal por primera vez. Estas plantas, en cuyas flores el cigarrón o "carpenter bee", Xylocopa brasilianorum L., encuentra alimento (néctar y polen) y en algunos casos, madera para sus nidos, se han investigado. Se sugiere que que el cigarrón inadvertida, directa o indirectamente, es responsable de la polinización de 25 ó más especies de frutas y semillas que constituyen una fuente de alimento para el hombre en Puerto Rico.

\section{LITERATURE CITED}

1. Barnés, D. K., and Freyre, R. H., Seed production potential of Tephrosia vogelii in Puerto Rico, J. Agr. Univ. P.R., 53 (2): 207-12, 1969.

2. Britton, N. L., and Wilson, P., Botany of Porto Rico and the Virgin Islands-Descriptive Flora-Spermatophyta, Sci. Surv. P.R. and the Virgin Islands., N.Y. Acad. Sci. 5: p 626., and 6: p 663., 1923-30.

3. Cabanillas, E., Personal communication, Mayagüez Inst. of Trop. Agr. Box 70, Mayagüez, P.R. 00807 USA.

4. Gundlach, J., Apuntes para la fauna Puerto-Riqueña, Anal. Soc. Esp. Hist. Nat., 26 (6): $154,1887$.

5. Jackson, George C., and Bauzá Salas, J., Insect visitors of Lecythis elliptica, H.B.K., J. Agr. Univ. P.R., 49 (1): 133-41, 1965.

6. Kapil, R. P., and Dhaliwal, J. S., Biology of Xylocopa species: II. Field activities, flight range and trials on transportation to nests, J. Res. Punjab. Agr. U., 6 (1): 262-71, 1969. 
7. Kelsey, Harlan P., and Dayton, William A., Standardized Plant Names, J. Horace McFarland Co., Harrisburg, Pa., 675 pp., 1942.

8. Lindsey, E. G., The ecology of solitary bees, Hilgardia, 27 (19): 543-99, 1958.

9. Liogier, Brother Alain H., Nomenclatural changes and additions to Britton and Wilson's "Flora of Puerto Rico and the Virgin Islands". Rhodora, 67 (722): 315-61, 1965.

10. Further changes and additions to the Flora of Puerto Rico and the Virgin Islands, Rhodora, 69 (779): 372-6, 1967.

11. Little, Elbert L., Jr., and Wadsworth, Frank H., Common trees of Puerto Rico and the Virgin Islands. USDA Agricultural Handbook 249, 548 pp., illus., 1964.

12. - Woodbury, Roy O., and Wadsworth, Frank H., Trees of Puerto Rico and the Virgin Islands, 2nd. volume. USDA Agriculture Handbook 449, 1024 pp., illus., 1974.

13. Longwood, F. R., Puerto Rican woods; their machining, seasoning and related characteristics, USDA, Agriculture Handbook 205, 98 pp. illus., 1961.

14. McClure, F. A., The bamboos, a fresh perspective. Harvard Univ. Press, Cambridge, Mass., 289-91. 1966.

15. Martin, F. W., Cabanillas, E., and Ortiz, S., Natural pollination, hand pollination and crossability of some Mexican species of Dioscorea, Trop. Agr. Trin., 40 (2): 135-41, 1963.

16. Martorell, Luis F., A survey of the forest insects of Puerto Rico: Part II, J. Agr. Univ. P.R., 29 (4): 355-608, 1945.

17. Nishida, T., Pollination of the passion fruit in Hawaii, J. Econ. Ent. 51 (2): 146-56, 1958.

18. Otero, J. I., Toro, R. A., and Pagán de Otero, Lydia, Catálogo de los nombres vulgares y cientifícos de algunas plantas puertorriqueñas, Est. Exp. Agr. Univ. P.R., Boletín 37, 1945.

19. Ramos, J. A., The insects of Mona Island (West Indies), J. Agr. Univ. P.R. 30 (1): 1$74,1946$.

20. Stahl, A., Catálogo del gabinete zoológico de Dr. A. Stahl, (Fauna de Puerto Rico), 249 pp., Imp. del "Boletın Mercantil", San Juan, P.R., 1882.

21. Wolcott, G. N., "Insectae Portoricensis”, J. Dept. Agr. P.R. 7 (1): 1-313, 1923.

22. —- Insectae Borinquenses, J. Agr. Univ. P.R., 20 (1): 572-3, 1936.

23. - - Supplement to "Insectae Borinquenses," J. Agr. Univ. P.R. 25 (2): 33-158, 1921.

24. Insects of Puerto Rico, J. Agr. Univ. P.R., 32 (4): 870-1, 1948. 